\title{
Enhanced signaling scheme with admission control in the hybrid optical wireless (HOW) networks
}

\author{
Yan, Ying; Yu, Hao; Wessing, Henrik; Dittmann, Lars
}

Published in:

IEEE INFOCOM Workshops 2009

Link to article, DOI:

10.1109/INFCOMW.2009.5072139

Publication date:

2009

Document Version

Publisher's PDF, also known as Version of record

Link back to DTU Orbit

Citation (APA):

Yan, Y., Yu, H., Wessing, H., \& Dittmann, L. (2009). Enhanced signaling scheme with admission control in the hybrid optical wireless (HOW) networks. In IEEE INFOCOM Workshops 2009 IEEE.

https://doi.org/10.1109/INFCOMW.2009.5072139

\section{General rights}

Copyright and moral rights for the publications made accessible in the public portal are retained by the authors and/or other copyright owners and it is a condition of accessing publications that users recognise and abide by the legal requirements associated with these rights.

- Users may download and print one copy of any publication from the public portal for the purpose of private study or research.

- You may not further distribute the material or use it for any profit-making activity or commercial gain

- You may freely distribute the URL identifying the publication in the public portal

If you believe that this document breaches copyright please contact us providing details, and we will remove access to the work immediately and investigate your claim. 


\title{
Enhanced Signaling Scheme with Admission Control in the Hybrid Optical Wireless (HOW) Networks
}

\author{
Ying Yan, Hao Yu, Henrik Wessing, and Lars Dittmann \\ Department of Photonics \\ Technical University of Denmark \\ Lyngby, Denmark \\ \{yiya, haoyu, hewe, ladit\}@fotonik.dtu.dk
}

\begin{abstract}
The hybrid optical wireless (HOW) network has been viewed as a promising solution to meet the increasing user bandwidth and mobility demands. Due to the basic differences in the optical and wireless technologies, a challenging problem lies in the Media Access Control (MAC) protocol design so that it can support stringent Quality of Service $(\mathrm{QoS})$ requirements. In this paper, we describe and evaluate a resource management framework designed for the HOW networks. There are two parts in the resource management framework The first part is the Enhanced MPCP (E-MPCP) scheme aiming at improving signaling ability between the optical and wireless networks. The second part is an Integrated Optical Wireless Admission Control (IOW-AC) scheme aiming at providing the integrated bandwidth allocation in the HOW networks. Our results show that the performances of the system in terms of throughput, delay and packet dropping probability depend on several factors. These factors include the frame duration, the traffic load and the total number of shared users. The results also highlight that our proposed system achieves significant improvements over the traditional approach in terms of user QoS guarantee and network resource utilization.
\end{abstract}

Keywords- call admission control, MPCP, resource allocation, the hybrid optical wireless network

\section{INTRODUCTION}

Recent years have witnessed the explosive growth of the multimedia applications, which are characterized with high speed and stringent QoS requirements. An integration of optical and wireless technologies has been viewed as a viable solution to delivery the quadruple play services (data, voice, video and mobility). In the HOW networks, complementary characteristics of the optical network and wireless network are combined. In optical access network, such as Ethernet Passive Optical Network (EPON), has been deployed to satisfy bandwidth demands for broadband services, but it has a fixed architecture and limited coverage. Among various available wireless technologies, the recent Worldwide
Interoperability for Microwave Access (WiMAX) technology has promised ubiquitous and high data rate connections for both fixed and mobile communication. A hybrid EPON and WiMAX network is an example of the HOW network, which is a convergence of an EPON deployed as a backhaul and multiple WiMAX networks connected as the front-end networks. Since the opticaland wireless-access technologies are originally designed to address different issues and deployed in different scenarios, any simple combination of them cannot derive optimal network performances. One major challenge in the HOW network is how to design a heterogeneous resource management framework, which can be exploited to maximize the overall resource utilization and optimize QoS performances in both the optical domain and the wireless domain simultaneously.

There are several aspects needed to be addressed in advance to achieve an efficient resource management. The main technical issues are as follows: (1) routing algorithm, (2) packet classification and scheduling and (3) resource management. First, the integrated routing algorithm has been proposed in [1,2] to select the optimum routes for transmissions in both optical and wireless domain. A risk- and delay- aware routing algorithm is proposed for the front-end wireless network in $[3,4]$. Second, scheduling algorithm is also improved to consider the network condition jointly in the optical and wireless domain [5]. In [6], we proposed a scheduler, which prioritizes connections in the optical domain, while taking wireless channel quality into account. At last, for the resource management, as proposed in [7], a central controller is designed in the optical domain to have a view of the hybrid network. The connection requests submitted in the wireless domain are delivered to the optical domain. As designed for the HOW network, a utility-based bandwidth allocation scheme is proposed in [8]. Capacity analysis and wavelength multiplexing are exploited in [9] for flexible bandwidth allocation. Although the idea of an integrated resource management is proposed, how to realize such scheme has 
not been addressed. In this paper, we presents our study and design of a resource management framework for the HOW networks. Our proposed scheme can jointly and optimally admit the request connections and allocate the network resources for both the optical network and the wireless network with an overall consideration. Under an integrated resource management framework, the potential network congestion is able to be avoided, the resource utilization can be maximized and the prescribed QoS requirements are able to be successfully satisfied.

The rest of this paper is organized as follows. Section II introduces our proposed resource management framework. Section III presents the simulation results and analysis. Finally, conclusion is given in Section IV.

\section{ENHANCED SignALLING SCHEME WITH AC}

\section{A. Hybrid EPON and WiMAX Network Architecture}

The hybrid EPON and WiMAX network architecture is illustrated in Figure 1. In EPON, a centralized Optical Line Terminal (OLT) communicates with multiple connected Optical Network Users (ONUs) via a passive optical splitter. In the Point-to-Multipoint (PMP) WiMAX network, a Base Station (BS) manages channel allocation to Subscriber Stations (SSs) as a central controller [14]. In hybrid network architecture, the ONU functions and BS functions are integrated into a single device, namely an Access Gateway $(A G)$, which handles connections within the wireless network, and connections cross both EPON and WiMAX network. The upstream traffic is first aggregated at an $A G$ and then forwarded to the OLT. For the downstream traffic, packets are first transmitted to the AG and then forwarded to each SS in the allocated channel slots.

Traditionally network control mechanisms are designed separately and exclusively for either optical

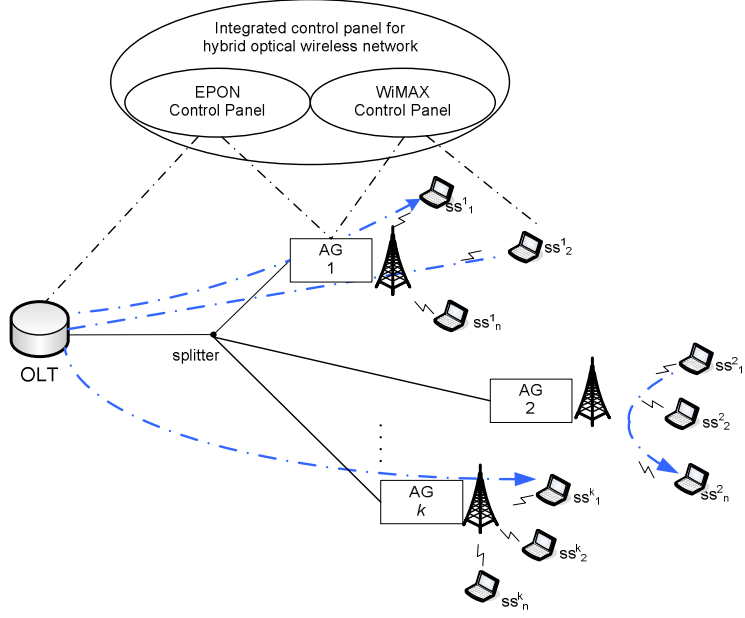

Figure 1. Integrated optical wireless network model networks or wireless networks. The characteristics and challenges of the HOW network are ignored. To achieve desired network performances, the existing control planes in EPON and WiMAX should be integrated and improved with advanced administration functions. Figure 2. shows an overview of operations implemented in the OLT and the AG for scheduling, resource allocation and admission control. In this paper, we propose a modified resource negotiation scheme at the optical uplink bandwidth allocator block in the OLT and a delay-aware admission control at the CAC block in the AG.

\section{B. Basic MPCP Operations}

A typical EPON system is a tree-based architecture, which consists of one Optical Line Terminal (OLT) functionalized as a central control station, one 1:N (for example, $N=16$ or 32) passive optical splitter and multiple Optical Network Users (ONUs). The framework of the EPON MAC layer, known as the MultiPoint Control Protocol (MPCP), is defined in IEEE 802.3ah Ethernet in the First Mile (EFM) Task Force [10]. MPCP is a signaling access protocol, which uses two 64-byte MAC control messages to register the connected ONUs and allocation upstream bandwidth among ONUs (via GATE and REPORT messages). Since the upstream transmissions from multiple ONUs to the OLT share a common optical link, an important issue in EPON is to efficiently allocate resources to multi-users, which is not specified in the standard.

Two control messages, REPORT and GATE, are defined in the MultiPoint Control Protocol (MPCP) as signaling between the OLT and AGs in the hybrid network. The REPORT message is in the downstream directed to the OLT and the GATE message is in the upstream initiated from the OLT. The OLT registers attached AGs within the discovery period, which is repeated periodically so that newly connected AGs can seamlessly be added without interrupting the current network operation. After discovering and registering the connected AGs, the OLT sets up an entry table, which contains the AG node identification (NID) and the round trip time $\left(T_{r t t}\right)$. Since multiple AGs share a common optical link in the upstream direction, the OLT requires assigning the bandwidth in a manner of fairness and without conflict. The upstream transmission period is divided into multiple time slots and each ONU can only transmit during its own slot time. In the normal processing state, the OLT polls registered AGs and assigns time slots either statically based on the TDMA scheme or dynamically based on the designed resource requirement negotiation [11-13]. In this paper, we use the TDMA in the EPON domain. In TDMA scheme, one AG is granted a fixed time slot length and is polled in order. In this paper, a cycle $\left(T_{\text {cycle }}\right)$ refers to a period in which all 


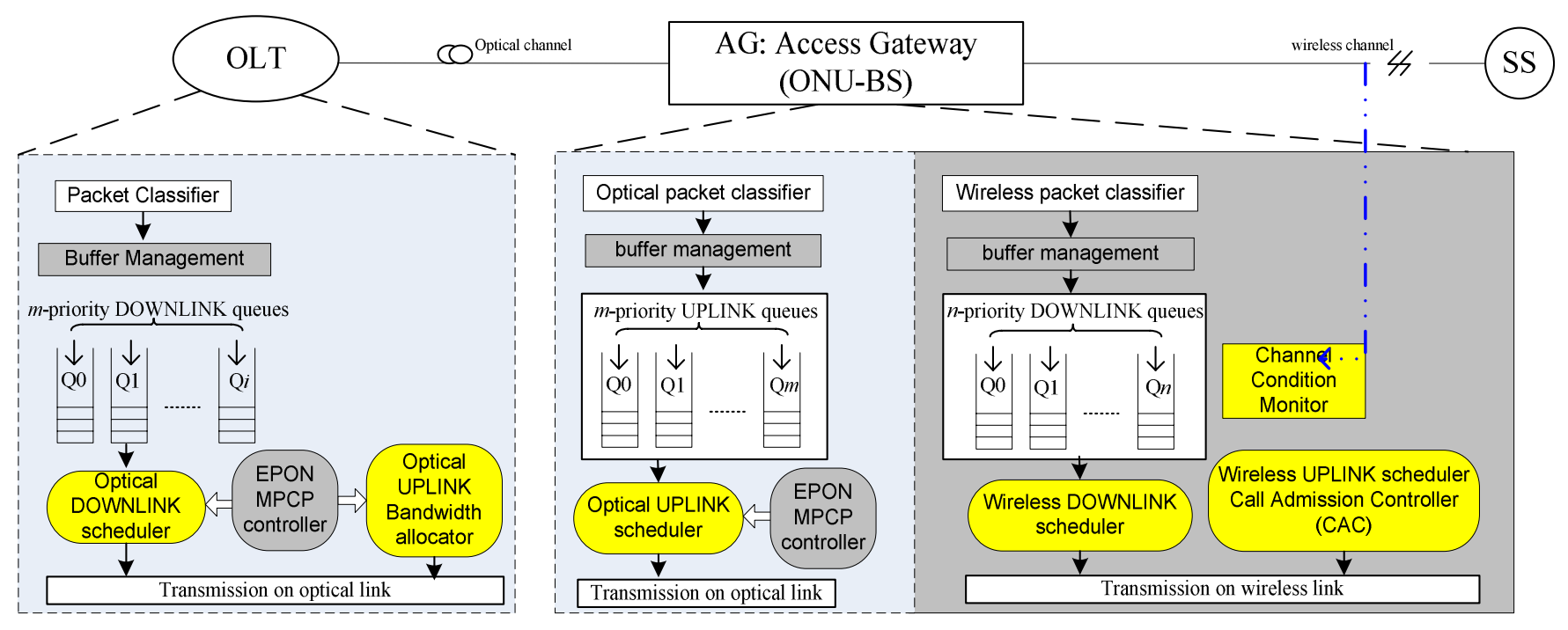

Figure 2. Functional overview of an integrated control framework

AGs are served and a subframe refers to a period, which is assigned to an AG for the uplink transmission.

\section{Enhanced MPCP Scheme}

In the integrated architecture, an AG performs resource management operations for both optical and wireless networks. In order to calculate the packet transmission delay in the optical domain, the AG needs to know the estimated waiting time for its next poll. This extra information can be derived from the OLT using a modified GATE message. Originally defined in MPCP, the GATE message consists of a 1-byte granted start time $\left(t_{\text {start }}\right)$ and a 2-byte granted bandwidth $(B W)$. We add a 3byte field, the next cycle time $\left(T_{\text {next }}\right)$, which is the interval between two adjacent polling operations to an AG.

After the OLT computes the start time and subframe length for each AG, the OLT can obtain the total transmission length $\left(T_{\text {cycle }}\right)$ for all $\mathrm{k}$ AGs. We use $t$ to indicate the time and $T$ to indicate the duration

$$
T_{\text {cycle }}=\sum_{i=0}^{k-1}\left(\frac{B W^{i}}{R_{o}}+T_{g}\right)
$$

where $R_{o}$ is the transmission rate of the optical uplink and $T_{g}$ is the guard time between two successive upstream transmissions.

After a period of $T_{\text {cycle }}$, an AG is polled again. The interval between two adjacent polling operations, the next cycle time, can be calculated and added into the original GATE message as a new field. The GATE control message can be embedded within an Ethernet frame. The receiver recognizes the GATE control message and then extracts the following 6-byte grant information. The enhanced MPCP scheme under the TDMA is shown in Figure 3. The polling sequence of AGs is scheduled in the OLT. The value of granted bandwidth is fixed and same to all $k$ AGs. In other words, each AG has to wait $T_{\text {cycle }}$ as the polling wait time. The value of $t_{\text {start }}$ and $T_{\text {cycle }}$ are updated and changed once there are new AGs registered and scheduled. The expected next cycle time for the $i^{\text {th }} \mathrm{AG}$ is calculated in Eq. (2). The entry table is updated after the periodic discovery process. The value of $t_{\text {next }}$ for each AG is computed at the beginning of each cycle.

$$
\begin{aligned}
t_{\text {next }}^{i}-T D M A & =t_{\text {start }}^{i}+T_{\text {cycle }} \\
& =t_{\text {start }}^{i}+k \cdot\left(\frac{B W}{R_{o}}+T_{g}\right)
\end{aligned}
$$

\section{Integrated Optical Wireless Admission Control}

A key characteristic of our proposed resource management is to provide overall considerations of both optical and wireless network conditions. Although EPON and WiMAX use different channel access mechanisms, they both need admission control to determine how much traffic can be handled in the optical and the wireless domains separately, so that the prescribed QoS for each traffic stream can be maintained. Implementing two individual admission control schemes for each network is not effective and efficient. An Integrated Optical Wireless Admission Control (IOW-AC) scheme is proposed to cope with this problem, which provides delay bounds to multidomain connections. We first present a model to evaluate the delays experienced by SSs and then describe the proposed IOW-AC scheme. 
When traffic arrives at the AG from the SSs, AG is able to estimate the waiting time for the traffic to be served. The overall estimated delay $\left(d_{e s t}\right)$ includes the waiting time until $\mathrm{AG}^{\mathrm{i}}$ is polled by the OLT $\left(d_{\text {polling }}\right)$, the waiting time for the prior data in the same queue being served $\left(d_{\text {queueing }}\right)$ and the wireless transmission $\left(d_{t x \text {-wireless }}\right)$ and propagation delay $\left(d_{\text {prop-wireless, }}\right)$.

$$
d_{\text {est }}=d_{\text {polling }}+d_{\text {queuing }}+d_{\text {tx-wireless }}+d_{\text {prop-wireless }}
$$

Where $d_{\text {polling }}$ is calculated based on the next cycle time $\left(t_{\text {next }}\right)$ and the packet arrival time $(t)$. The $t_{\text {next }}$ is computed as we explained earlier. $d_{\text {queueing }}$ is determined by the current subqueue size and the assigned bandwidth within a subframe. If the subframe has enough empty space, the new coming packet can be served right after the queued packets in the following subframe. Since the queuing delay is not our focus, we assume the available buffer size in AG is enough. $d_{t x \text {-wireless }}$ is calculated based on the packet size and the wireless link rate.

$d_{\text {polling }}$ is determined by the information (e.g. the starting transmission time $t_{0}$, the granted slot time $t_{\text {granted }}$ and the next transmission time $t_{\text {next }}$ ) provided in the GATE message. In the case $t \in\left[t_{0}^{i}, t_{0}^{i}+T_{\text {granted }}^{i}\right.$, the request arrives at the time when the AG is being polled. the request of $j^{\text {th }} \mathrm{SS}, \mathrm{SS}^{\mathrm{j}}$, arrives when the $\mathrm{AG}^{\mathrm{i}}$ is being polled. A request can be accepted and served within the current subframe only if the corresponding packets can be received before the current subframe is finished. Thus, $d_{\text {polling }}=0$. Otherwise, $d_{\text {polling }}=t_{\text {next }}-t$. If the request of SS arrives at the AG, which is waiting to be polled, i.e., $t \in[$ $t_{0}+T_{\text {granted, }}^{i} t_{0}+T_{\text {next-tx } x}^{i}$ ), the packet has to wait until the remaining part of an uplink transmission from other AGs to the OLT is completed. The earliest time for the request

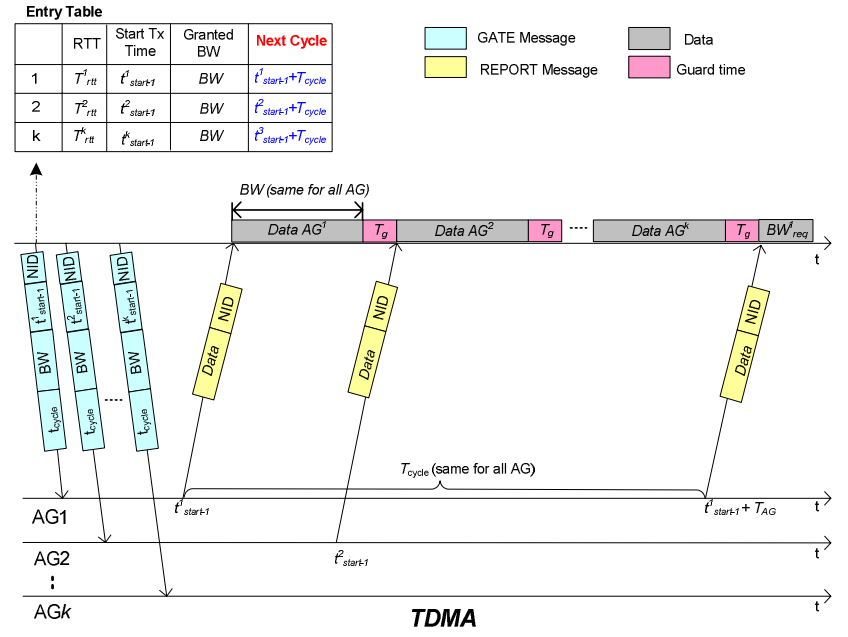

Figure 3. Enhanced MPCP with TDMA scheme to get served is the next polling time. Thus, $d_{\text {polling }}=t_{\text {next }}-t$ It is noted that an increase on the subframe size $T_{\text {granted }}$ can result in a larger number of accepted requests, but it also increases the polling delay.

In order to support and protect the QoS of real-time traffic streams, in addition to bandwidth allocation, an AC scheme is required to decide whether to admit a realtime traffic stream based on both admission policies and QoS requirements supplied by the application at the end users. Assuming for real-time services, QoS metrics are predefined and various thresholds are specified. The realtime traffic flows are characterized by the demand QoS parameters, for example, the delay bound $\left(d_{\text {min }}\right)$. The AG can perform rate-based $\mathrm{AC}$ that is based on the delay requirement of the new arriving flow and the estimated delay. The IOW-AC provides guaranteed QoS and increases the network throughput by accepting requests without violating the delay constraints. The proposed IOW-AC scheme can be accomplished as follows. As illustrated in Figure 4., when there is a new request received at $\mathrm{AG}$. The transmission and propagation delay are first calculated according to the wireless network

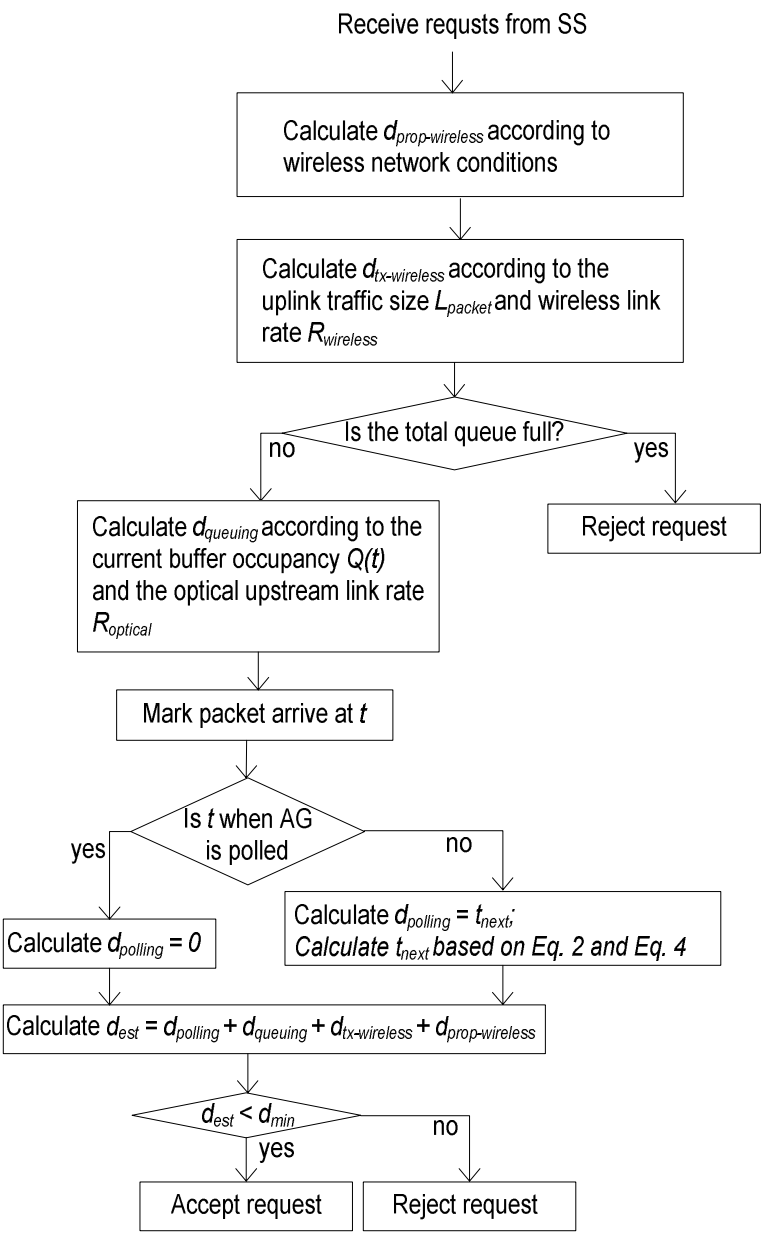

Figure 4. Operations of the proposed uplink IOW-AC scheme. 
conditions and traffic profile. Secondly, the queuing delay is evaluated based on the current buffer occupancy. Then, using the polling status information, the expected polling delay is computed as explained in Eq. (1) and Eq. (2). Finally, when the IOW-AC approach determines the overall delay, $d_{e s t}$, for a multidomain connection request, it compares the delay with the delay bound, $d_{m i n}$. If the required delay bound is satisfied, the request is accepted $\left(d_{e s t}<d_{\min }\right)$. Otherwise, the request is rejected.

\section{PERFORMANCE EVAluAtion}

Extensive simulations have been conducted using OPNET modeler [15] to evaluate the performance of the proposed enhanced MPCP and IOW-AC scheme for the HOW network. Simulation results are shown to compare the proposed framework with the traditional separated resource management schemes. Simulations are carried out for different network sizes and traffic loads.

The total number of AGs $K$ is 32 and the EPON link rate is assumed as $1 \mathrm{~Gb} / \mathrm{s}$. The guard time between two adjacent transmissions on the optical uplink fiber is $5 \mu$ s. We assume that the AG supports a $70 \mathrm{Mb} / \mathrm{s}$ WiMAX uplink data rate. Based on the IEEE 802.16 standard, both rtPS and BE traffic are considered. For users of rtPS traffic, e.g. video streaming service, the traffic profile has a variable packet size, uniformly distributed between 1200 and 1500 bytes. The delay bound for the real time traffic is set as $75 \mathrm{~ms}$.

Figure 5a. shows the ratio of admitted requests in the total received requests for both real-time traffic and $\mathrm{BE}$ traffic, using the two different AC schemes. The incoming requests can be admitted only when their QoS requirements are met. This figure shows that the IOW$\mathrm{AC}$ admitted larger amount of conforming traffic than the Normal-AC for both the real-time and BE traffic. The differences become more significant when the traffic arrival rate increases. The channel utilization becomes better in the IOW-AC because there is wastage in the Normal-AC scheme taken by the nonconforming traffic.

In Figure $5 b$ we compare the simulation results for the dropping probability for the real-time traffic and $\mathrm{BE}$ traffic when there is limited buffer size at an AG node. IOW-AC scheme achieves lower dropping probability for both real time traffic and best effort (BE) traffic. This is because that IOW-AC only accepts conforming traffic and saves more bandwidth for the conforming real time traffic without any drop even under high real-time traffic rate. We observe the similar result, which is that IOWAC yields better bandwidth utilization.

In Figure $5 \mathrm{c}$ we plot the percentage of accepted requests for different length of subframe period ranging from $1 \mathrm{~ms}$ to $5 \mathrm{~ms}$. As we mentioned before, when the subframe period $\left(T_{\text {granted }}\right)$ increases, there are more requests can be served in the uplink optical link. However, the total cycle time $\left(T_{\text {cycle }}\right)$ becomes larger and the polling delay increases simultaneously. In this figure, the IOW-AC scheme blocks nonconforming traffic especially when the subframe period is large. Under the Normal-AC scheme, the accepted real-time traffic is most of nonconforming traffic.

In Figure $6 \mathrm{a}$ we examine the polling delay $\left(d_{\text {polling }}\right)$ experienced at the AG node in the IOW-AC scheme. The buffer size is specified as unlimited. Therefore, the $T_{\text {queuing }}$ of voice and video traffic will not be a dominating
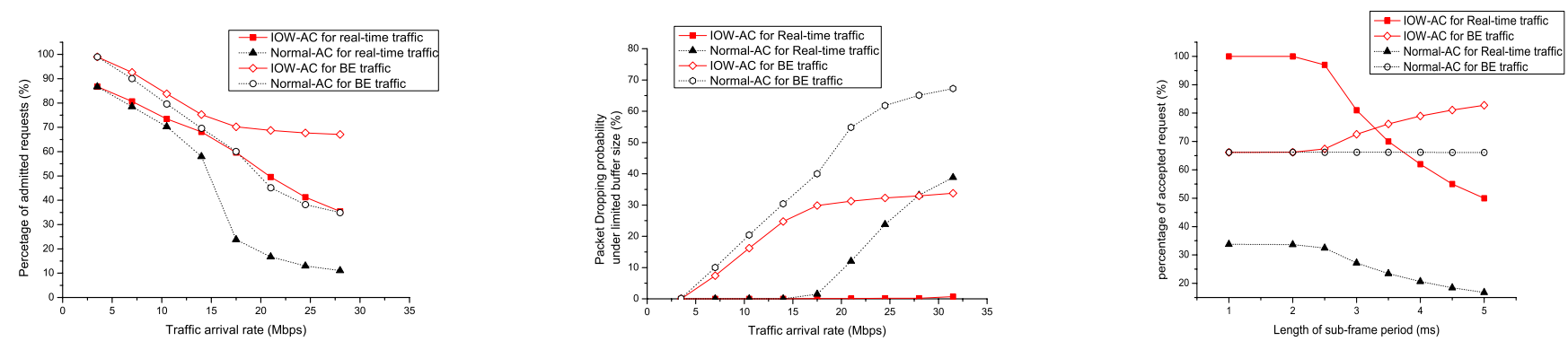

Figure 5. (a) Admitted request Vs. traffic arrival rate. (b) Packet dropping probability Vs. traffic rate. (c) Admitted request Vs. subframe length
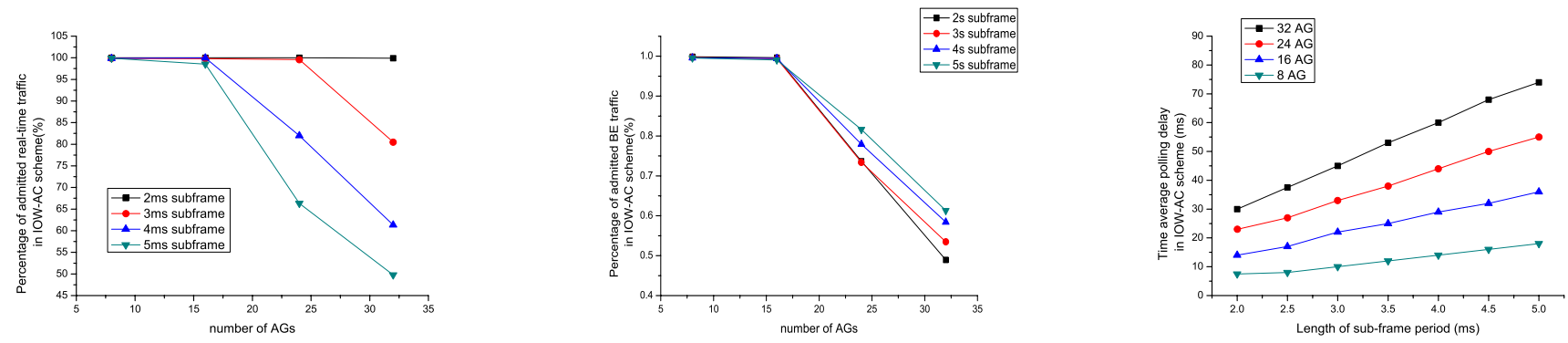

Figure 6. (a) Polling delay Vs. subframe length. (b) Admitted RT request Vs. number of AGs. (c) Admitted BE request Vs. number of AGs 
factor in the AC. On the contrary, the influence of $T_{\text {polling }}$ on $\mathrm{AC}$ is highlighted, which is important to observe in simulations. The value of the polling delay is zero if the request packet is received during the subframe period. Otherwise, the polling delay is the interval between the current time and the next poll time. In this figure, the polling delay is a time average value. As the subframe period increases, the polling delay becomes larger because it takes longer time for the OLT to serve all AGs. Therefore, the polling delay is also depending on the number of connected AGs. The more the number of $A G$, the longer the polling delay.

In Figure $6 \mathrm{~b}$ and Figure $6 \mathrm{c}$ we compare admitted realtime traffic and the BE traffic under different number of AGs and different length of subframes, in the IOW-AC scheme. The number of blocked requests is increased using the IOW-AC scheme when the number of AGs and the period of subframe become larger. We have observed that the benefit of our proposed integrated resource management framework is highly depends on the network size and traffic profile.

\section{CONCLUSION}

In this paper we present an integrated resource management framework for the hybrid optical wireless networks aiming for maximum user QoS and maximum network throughput. Simulations conducted using the OPNET modeler show that the proposed system achieves significant improvements over the traditional admission control approach in terms of user QoS guarantee and network resource utilization. These gains are achieved without complex modification on the existed network protocols. Another interesting observation is that if the number of AGs and the assigned subframe period for each AG are increased, the proposed admission control scheme achieves more significant improvements. Since the network throughput is relevant to the AG parameters, an operator can estimate the performance based on this simulation results.

The benefit of our proposed integrated resource management framework highly depends on the network size and traffic profile. The analysis could be a good assistance or guidance in future research in many fields of hybrid optical wireless network architecture, including resource management, optimizing QoS and demanding service provisioning.

\section{ACKNOWLEDGMENT}

This work was supported in part by the European Committee through project ALPHA (Architectures for fLexible Photonic Home and Access networks).

\section{REFERENCES}

[1] W.-T. Shaw, D. Gutierrez, K. S. Kim, N. Cheng, S.-H. Wong, S.-H. Yen, and L. G. Kazovsky, "GROW-Net-A hybrid optical wireless access network," in Proc. 9th Joint Conf. Information Sciences. (Invited Paper), Oct. 2006.

[2] W.-T. Shaw, S.-H. Wong, N. Cheng, K. Balasubramanian, X. Zhu, M. Maier and L. G. Kazovsky, "Hybrid architecture and integrated routing in a scalable optical-wireless access network," Journal of Lightwave Technology, Vol. 25, No. 11, Nov. 2007.

[3] S. Sarkar, S. Dixit and B. Mukherjee, "Hybrid wireless-optical broadband-access network (WOBAN): a review of relevant challenges", Journal of Lightwave Technology (invited paper), Vol. 25, No.11, Nov. 2007.

[4] S. Sarkar, H. Yen, S. Dixit and B. Mukherjee, "DARA: delayaware routing algorithm in a hybrid wireless-optical broadband access network (WOBAN)", in Proc. IEEE International Conference on Communications, Jun. 2007.

[5] G. Shen, R. S. Tucker and C. Chae, "Fixed mobile convergence architectures for broadband access: integration of EPON and WiMAX", IEEE Communications Magazine, pp. 44-50, Aug. 2007.

[6] Y. Yan, H. Yu and L. Dittmann. "Wireless channel condition aware scheduling algorithm for hybrid optical/wireless networks", in Proc. 3rd International Conference on Access Networks, Oct. 2008.

[7] Y. Luo, S. Yin, T. Wang, Y. Suemura, S. Nakamura and M. Cvijectic, "QoS-aware scheduling over hybrid optical wireless networks", in Proc. Optical Fiber Communication Conference and National Fiber Optic Engineers Conf., 2006, pp 1-7.

[8] P. Lin, C. Qiao, T. Wang and J. Hu. "Optimal utility-based bandwidth allocation over integrated optical and WiMAX networks", in Proc. Optical Fiber Communication Conference and National Fiber Optic Engineers Conf., 2006, pp 3.

[9] H. Kim and A. Wolisz. "A radio over fiber based wireless access network architecture for rural areas", in Proc. $14^{\text {th }}$ IST Mobile and Wireless Communications Summit, Jun. 2005.

[10] Ethernet in the First Mile Alliance (EFMA), "Ethernet Passive Optical Network (EPON) tutorial", Revision 4, (2004).

[11] Kramer Glen, Mukherjee Biswanath, and Pesavento Gerry. "IPACT: a dynamic protocol for an Ethernet PON (EPON)," IEEE Communication Magazine 40(2), 74-80 (2002).

[12] C. Assi et al., "Dynamic bandwidth allocation for quality-ofservice over Ethernet PON," IEEE JSAC, vol. 12, no. 9, pp. 1467-77 (2003).

[13] M. Ma, Y. Zhu, and T. H. Cheng, "A systematic scheme for multiple access in ethernet passive optical access networks," J. Lightwave Tech., vol. 23, no. 11, pp. 3671-82 (2005).

[14] Jeffrey G. Andrews, Arunabha Ghosh and Rias Muhamed, [Fundamentals of WiMAX], Prentice Hall (2007).

[15] OPNET Modeler 14.0, http://www.opnet.com/. 